Pacific

Journal of

Mathematics

TWISTED MODULES FOR VERTEX ALGEBRAS ASSOCIATED WITH VERTEX ALGEBROIDS

HAISHENG Li AND GAYWALEE YAMSKULNA

Volume 229 No. 1

January 2007 


\title{
TWISTED MODULES FOR VERTEX ALGEBRAS ASSOCIATED WITH VERTEX ALGEBROIDS
}

\author{
HAISHENG LI AND GAYWALEE YAMSKULNA
}

\begin{abstract}
We continue the work in our earlier paper, "On certain vertex algebras and their modules associated with vertex algebroids", J. Algebra 283 (2005), 367-398, to construct and classify graded simple twisted modules for the $\mathbb{N}$ graded vertex algebras constructed by Gorbounov, Malikov and Schechtman from vertex algebroids. In addition, we determine the full automorphism groups of those $\mathbb{N}$-graded vertex algebras in terms of the automorphism groups of the corresponding vertex algebroids.
\end{abstract}

\section{Introduction}

For most of the important examples of vertex operator algebras $V=\bigsqcup_{n \in \mathbb{Z}} V_{(n)}$ graded by the $L(0)$-weight [Frenkel et al. 1988], the $\mathbb{Z}$-grading satisfies the condition $V_{(n)}=0$ for $n<0$ and $V_{(0)}=\mathbb{C} \mathbf{1}$, where $\mathbf{1}$ is the vacuum vector. For a vertex operator algebra $V$ with this special property, the homogeneous subspace $V_{(1)}$ has a natural Lie algebra structure with $[u, v]=u_{0} v$ for $u, v \in V_{(1)}$ and the product $u_{1} v\left(\in V_{(0)}\right)$ defines a symmetric invariant bilinear form on $V_{(1)}$.

A series of articles on gerbes of chiral differential operators [Gorbounov et al. 2004] and on chiral de Rham complexes [Malikov et al. 1998; 1999] have investigated $\mathbb{N}$-graded vertex algebras $V=\bigsqcup_{n \in \mathbb{N}} V_{(n)}$ with $V_{(0)}$ not necessarily 1dimensional. In this case, the bilinear operations $(u, v) \mapsto u_{i} v$ for $i \geq 0$ are closed on $V_{(0)} \oplus V_{(1)}$ :

$$
u_{i} v \in V_{(0)} \oplus V_{(1)} \quad \text { for } u, v \in V_{(0)} \oplus V_{(1)}, i \geq 0 .
$$

The skew symmetry and the Jacobi identity for the vertex algebra $V$ give rise to several compatibility relations. Such algebraic structures on $V_{(0)} \oplus V_{(1)}$ are summarized in the notion of what became known as a 1-truncated conformal algebra. The subspace $V_{(0)}$ equipped with the product $(a, b) \mapsto a_{-1} b$ is a commutative associative algebra with the vacuum vector $\mathbf{1}$ as the identity and $V_{(0)}$ as a nonassociative algebra acts on $V_{(1)}$ by $a \cdot u=a_{-1} u$ for $a \in V_{(0)}, u \in V_{(1)}$. All these structures on

MSC2000: primary 17B69; secondary 17B67.

Keywords: vertex algebra, twisted module, vertex algebroid.

Li was partially supported by an NSA grant. 
$V_{(0)} \oplus V_{(1)}$ are further summarized in the notion known as a vertex $A$-algebroid, where $A$ is a (unital) commutative associative algebra.

Gorbounov, Malikov and Schechtman also constructed in [Gorbounov et al. 2004], starting from any vertex $A$-algebroid, an $\mathbb{N}$-graded vertex algebra

$$
V=\coprod_{n \in \mathbb{N}} V_{(n)}
$$

such that $V_{(0)}=A$ and the vertex $A$-algebroid $V_{(1)}$ is isomorphic to the given one. All the $\mathbb{N}$-graded vertex algebras constructed are generated by $V_{(0)} \oplus V_{(1)}$ with a spanning property of PBW type. That work demonstrated that such $\mathbb{N}$ graded vertex algebras are natural and important to study. For example, the vertex (operator) algebra associated with a $\beta \gamma$ system, which plays a central role in free field realization of affine Lie algebras (see [Wakimoto 1986; Fer̆gin and Frenkel 1988; 1990a; 1990b, Frenkel and Ben-Zvi 2001]), is such an $\mathbb{N}$-graded vertex algebra. The vertex (operator) algebras constructed from toroidal Lie algebras are also of this type [Berman et al. 2002a; 2002b].

In [Li and Yamskulna 2005], we revisited those $\mathbb{N}$-graded vertex algebras and we classified all the $\mathbb{N}$-graded simple modules in terms of simple modules for certain Lie algebroids. In the theory of vertex algebras, in addition to the notion of module we have the notion of twisted module and twisted modules play a very important role, especially in the study of the so-called orbifold theory. Certainly, twisted modules also play an important role in other studies. In this paper, we continue to study the twisted modules for the $\mathbb{N}$-graded vertex algebras associated with vertex algebroids.

Let $B$ be a vertex $A$-algebroid and let $V_{B}$ be the associated $\mathbb{N}$-graded vertex algebra. In this paper, we define a notion of automorphism of the vertex $A$ algebroid $B$ and we prove that any automorphism of the vertex $A$-algebroid $B$ can be extended uniquely to an automorphism of the $\mathbb{N}$-graded vertex algebra $V_{B}$ and that the full automorphism group of the $\mathbb{N}$-graded vertex algebra $V_{B}$ is naturally isomorphic to the full automorphism group of the vertex $A$-algebroid $B$. Let $g$ be an automorphism of the vertex $A$-algebroid $B$ of order $T$ (finite). Then the $g$-fixed point $A^{0}$ is a subalgebra of $A$ and the $g$-fixed point $B^{0}$ is a vertex $A^{0}$-algebroid. Furthermore, $B^{0} / A^{0} \partial A^{0}$ is a Lie $A^{0}$-algebroid. It is proved that the category of $\frac{1}{T} \mathbb{N}$-graded simple $g$-twisted $V_{B}$-modules is equivalent to a subcategory of simple modules for the Lie $A^{0}$-algebroid $B^{0} / A^{0} \partial A^{0}$.

This paper is organized as follows: In the next section we review the construction of vertex algebras associated with vertex algebroids and we identify their automorphism groups with the automorphism groups of the vertex algebroids. In Section 3 we classify graded simple twisted modules. 


\section{Preliminaries}

We recall the notions of 1-truncated conformal algebra, vertex algebroid and Lie algebroid, and we review the construction of the $\mathbb{N}$-graded vertex algebra $V_{B}$ associated with a vertex $A$-algebroid $B$. We also define notions of (endomorphism) automorphism of a 1-truncated conformal algebra and of a vertex $A$-algebroid $B$. We then identify the group of grading-preserving automorphisms of $V_{B}$ with the group of automorphisms of the vertex $A$-algebroid $B$.

First, we recall from [Gorbounov et al. 2004] (compare [Bressler 2002; 2003]) the notions of 1-truncated conformal algebra, vertex algebroid and Lie algebroid.

Definition 2.1. A 1-truncated conformal algebra is a graded vector space $C=C_{0} \oplus$ $C_{1}$ equipped with a linear map $\partial: C_{0} \rightarrow C_{1}$ and bilinear operations $(u, v) \mapsto u_{i} v$ for $i=0,1$ of degree $-i-1$ on $C$ such that the following axioms hold:

1. (Derivation) for $a \in C_{0}, u \in C_{1}$,

$$
(\partial a)_{0}=0 ; \quad(\partial a)_{1}=-a_{0} ; \quad \partial\left(u_{0} a\right)=u_{0} \partial a
$$

2. (Commutativity) for $a \in C_{0}, u, v \in C_{1}$,

$$
u_{0} a=-a_{0} u ; \quad u_{0} v=-v_{0} u+\partial\left(v_{1} u\right) ; \quad u_{1} v=v_{1} u
$$

3. (Associativity) for $\alpha, \beta, \gamma \in C_{0} \oplus C_{1}$,

$$
\alpha_{0} \beta_{i} \gamma=\beta_{i} \alpha_{0} \gamma+\left(\alpha_{0} \beta\right)_{i} \gamma .
$$

Remark 2.2. Let $C=C_{0} \oplus C_{1}$ be a 1-truncated conformal algebra and let $\ell$ be any nonzero complex number. Set $C[\ell]=C_{0} \oplus C_{1}$ as a vector space. We retain all the structures on $C$ except that we change the bilinear operation $C_{1} \times C_{1} \rightarrow C_{0}$ : $u \times v \mapsto u_{1} v$ by multiplying $1 / \ell$ and change the linear operator $\partial$ by multiplying $\ell$. Then one can show that $C[\ell]$ is a 1-truncated conformal algebra.

Definition 2.3. Let $A$ be a unital commutative associative algebra over $\mathbb{C}$. A vertex $A$-algebroid is a $\mathbb{C}$-vector space $\Gamma$ equipped with

1. a $\mathbb{C}$-bilinear map

$$
A \times \Gamma \rightarrow \Gamma ; \quad(a, v) \mapsto a * v
$$

such that $1 * v=v$ for $v \in \Gamma$,

2. a Leibniz $\mathbb{C}$-algebra structure $[\cdot, \cdot]: \Gamma \otimes_{\mathbb{C}} \Gamma \rightarrow \Gamma$,

3. a homomorphism of Leibniz $\mathbb{C}$-algebras $\pi: \Gamma \rightarrow \operatorname{Der}(A)$,

4. a symmetric $\mathbb{C}$-bilinear pairing $\langle\cdot, \cdot\rangle: \Gamma \otimes_{\mathbb{C}} \Gamma \rightarrow A$.

5. a $\mathbb{C}$-linear map $\partial: A \rightarrow \Gamma$ such that $\pi \circ \partial=0$. 
All the following conditions are assumed to hold, for $a, a^{\prime} \in A, u, v, v_{1}, v_{2} \in \Gamma$ :

$$
\begin{aligned}
a *\left(a^{\prime} * v\right)-\left(a a^{\prime}\right) * v & =\pi(v)(a) * \partial\left(a^{\prime}\right)+\pi(v)\left(a^{\prime}\right) * \partial(a), \\
{[u, a * v] } & =\pi(u)(a) * v+a *[u, v], \\
{[u, v]+[v, u] } & =\partial(\langle u, v\rangle), \\
\pi(a * v) & =a \pi(v), \\
\langle a * u, v\rangle & =a\langle u, v\rangle-\pi(u)(\pi(v)(a)), \\
\pi(v)\left(\left\langle v_{1}, v_{2}\right\rangle\right) & =\left\langle\left[v, v_{1}\right], v_{2}\right\rangle+\left\langle v_{1},\left[v, v_{2}\right]\right\rangle, \\
\partial\left(a a^{\prime}\right) & =a * \partial\left(a^{\prime}\right)+a^{\prime} * \partial(a), \\
{[v, \partial(a)] } & =\partial(\pi(v)(a)), \\
\langle v, \partial(a)\rangle & =\pi(v)(a),
\end{aligned}
$$

Proposition 2.4 [Li and Yamskulna 2005]. Let A be a unital commutative associative algebra and let $B$ be a module for $A$ as a nonassociative algebra. Then a vertex $A$-algebroid structure on $B$ is equivalent to a 1 -truncated conformal algebra structure on $C=A \oplus B$ with

$$
\begin{aligned}
& a_{i} a^{\prime}=0, \\
& u_{0} v=[u, v], \\
& u_{1} v=\langle u, v\rangle, \\
& u_{0} a=\pi(u)(a), \\
& a_{0} u=-u_{0} a=-\pi(u)(a),
\end{aligned}
$$

for $a, a^{\prime} \in A, u, v \in B, i=0,1$, such that

$$
\begin{aligned}
a\left(a^{\prime} u\right)-\left(a a^{\prime}\right) u & =\left(u_{0} a\right) \partial a^{\prime}+\left(u_{0} a^{\prime}\right) \partial a, \\
u_{0}(a v)-a\left(u_{0} v\right) & =\left(u_{0} a\right) v, \\
u_{0}\left(a a^{\prime}\right) & =a\left(u_{0} a^{\prime}\right)+\left(u_{0} a\right) a^{\prime}, \\
a_{0}\left(a^{\prime} v\right) & =a^{\prime}\left(a_{0} v\right), \\
(a u)_{1} v & =a\left(u_{1} v\right)-u_{0} v_{0} a, \\
\partial\left(a a^{\prime}\right) & =a \partial\left(a^{\prime}\right)+a^{\prime} \partial(a) .
\end{aligned}
$$

Definition 2.5. Let $A$ be a unital commutative associative algebra. A Lie Aalgebroid is a Lie algebra $\mathfrak{g}$ equipped with an $A$-module structure and a module action on $A$ by derivation such that

$$
\begin{aligned}
{[u, a v] } & =a[u, v]+(u a) v, \\
a(u b) & =(a u) b
\end{aligned}
$$


for $u, v \in \mathfrak{g}$ and $a, b \in A$. A module for a Lie $A$-algebroid $\mathfrak{g}$ is a vector space $W$ equipped with a $\mathfrak{g}$-module structure and an $A$-module structure such that

$$
\begin{aligned}
& u(a w)-a(u w)=(u a) w, \\
& a(u w)=(a u) w
\end{aligned}
$$

for $a \in A, u \in \mathfrak{g}, w \in W$.

Lemma 2.6 [Bressler 2003]. Let $A$ be a unital commutative associative algebra (over $\mathbb{C}$ ) and let $B$ be a vertex A-algebroid. Then $B / A \partial A$ is naturally a Lie Aalgebroid.

Next, we recall the construction of vertex algebras associated with vertex algebroids, following the exposition of [Li and Yamskulna 2005].

First, starting with a 1-truncated conformal algebra $C=A \oplus B$ we construct a Lie algebra. Set

$$
L(A \oplus B)=(A \oplus B) \otimes \mathbb{C}\left[t, t^{-1}\right] .
$$

In the obvious way we define the subpaces $L(A)$ and $L(B)$. Set

$$
\hat{\partial}=\partial \otimes 1+1 \otimes d / d t: L(A) \rightarrow L(A \oplus B) .
$$

We define

$$
\begin{array}{ll}
\operatorname{deg}\left(a \otimes t^{n}\right)=-n-1 & \text { for } a \in A, n \in \mathbb{Z}, \\
\operatorname{deg}\left(b \otimes t^{n}\right)=-n & \text { for } b \in B, n \in \mathbb{Z},
\end{array}
$$

making $L(A \oplus B)$ a $\mathbb{Z}$-graded vector space. The linear map $\hat{\partial}$ is homogeneous of degree 1 . Set

$$
\mathscr{L}=L(A \oplus B) / \hat{\partial} L(A) .
$$

Define a bilinear product $[\cdot, \cdot]$ on $L(A \oplus B)$ such that for $a, a^{\prime} \in A, b, b^{\prime} \in B$, $m, n \in \mathbb{Z}$,

$$
\begin{aligned}
{\left[a \otimes t^{m}, a^{\prime} \otimes t^{n}\right] } & =0, \\
{\left[a \otimes t^{m}, b \otimes t^{n}\right] } & =a_{0} b \otimes t^{m+n}, \\
{\left[b \otimes t^{n}, a \otimes t^{m}\right] } & =b_{0} a \otimes t^{m+n}, \\
{\left[b \otimes t^{m}, b^{\prime} \otimes t^{n}\right] } & =b_{0} b^{\prime} \otimes t^{m+n}+m\left(b_{1} b^{\prime}\right) \otimes t^{m+n-1} .
\end{aligned}
$$

Proposition 2.7 [Li and Yamskulna 2005]. Let $C=A \oplus B$ be a 1-truncated conformal algebra. The subspace $\hat{\partial} L(A)$ of the nonassociative algebra $(L(A \oplus B),[\cdot, \cdot])$ is a two-sided ideal. The quotient nonassociative algebra $\mathscr{L}$ is a $\mathbb{Z}$-graded Lie algebra. 
Let $\rho$ be the projection map from $L(A \oplus B)$ to $\mathscr{L}$. For $u \in A \oplus B$ and $n \in \mathbb{Z}$, set

$$
u(n)=\rho\left(u \otimes t^{n}\right)=u \otimes t^{n}+\hat{\partial} L(A) \in \mathscr{L} .
$$

We have graded Lie subalgebras

$$
\begin{aligned}
& \mathscr{L}^{\geq 0}=\rho((A \oplus B) \otimes \mathbb{C}[t]), \\
& \mathscr{L}^{<0}=\rho\left((A \oplus B) \otimes t^{-1} \mathbb{C}\left[t^{-1}\right]\right)
\end{aligned}
$$

and we have $\mathscr{L}=\mathscr{L}^{\geq 0} \oplus \mathscr{L}^{<0}$ as a vector space.

Considering $\mathbb{C}$ as a trivial $\mathscr{L}^{\geq 0}$-module we form the induced module

$$
V_{\mathscr{L}}=U(\mathscr{L}) \otimes_{U(\mathscr{L} \geq 0)} \mathbb{C} .
$$

We assign $\operatorname{deg} \mathbb{C}=0$, making $V_{\mathscr{L}}$ naturally an $\mathbb{N}$-graded $\mathscr{L}$-module:

$$
V_{\mathscr{L}}=\coprod_{n \in \mathbb{N}}\left(V_{\mathscr{L}}\right)_{(n)} .
$$

Throughout this paper, $\mathbb{N}$ denotes the set of nonnegative integers. Set

$$
\mathbf{1}=1 \otimes 1 \in V_{\mathscr{L}} .
$$

By the P-B-W theorem, we have $V_{\mathscr{L}}=U\left(\mathscr{L}^{<0}\right)=S\left(\mathscr{L}^{<0}\right)$. In view of this, we can and we do consider $A \oplus B$ as a subspace:

$$
A \oplus B \rightarrow V_{\mathscr{L}} ; \quad u \mapsto u(-1) \mathbf{1} .
$$

Theorem 2.8 ([Li and Yamskulna 2005]; compare [Dong et al. 2002]). There exists a unique vertex algebra structure on $V_{\mathscr{L}}$ with $\mathbf{1}$ as the vacuum vector and with $Y(u, x)=\sum_{n \in \mathbb{Z}} u(n) x^{-n-1}$ for $u \in A \oplus B$. Moreover, the vertex algebra $V_{\mathscr{L}}$ is naturally an $\mathbb{N}$-graded vertex algebra and is generated by the subspace $A \oplus B$ with A of degree 0 and $B$ of degree 1.

Remark 2.9. For $n \in \mathbb{Z}$, set

$$
A(n)=\{a(n) \mid a \in A\}, \quad B(n)=\{b(n) \mid b \in B\} \subset \mathscr{L},
$$

and we set

$$
B(-)=\coprod_{n=1}^{\infty} B(-n) \subset \mathscr{L} .
$$

Both $A(-1)$ and $B(-)$ are Lie subalgebras of $\mathscr{L}^{<0}$ and we have $\mathscr{L}^{<0}=A(-1) \oplus$ $B(-)$ as a vector space. Then

$$
V_{\mathscr{L}}=U\left(\mathscr{L}^{<0}\right)=S\left(\mathscr{L}^{<0}\right)=S(A(-1) \oplus B(-))=S(B(-)) \otimes S(A(-1)) .
$$

Consequently, $\left(V_{\mathscr{L}}\right)_{(n)}=S(B(-))_{(n)} \otimes S(A(-1))$ for $n \in \mathbb{N}$. In particular, $\left(V_{\mathscr{L}}\right)_{(0)}=$ $S(A(-1))$. 
Now, we assume that $A$ is a unital commutative associative algebra with identity $e$ and that $B$ is a vertex $A$-algebroid. Thus $C=A \oplus B$ is a 1-truncated conformal algebra. We set

$$
\begin{aligned}
E & =\operatorname{span}\left\{e-\mathbf{1}, a(-1) a^{\prime}-a a^{\prime}, a(-1) b-a b \mid a, a^{\prime} \in A, b \in B\right\} \subset V_{\mathscr{L}}, \\
I_{B} & =U(\mathscr{L}) \mathbb{C}[\mathscr{D}] E .
\end{aligned}
$$

It was proved in [Li and Yamskulna 2005] that the $\mathscr{L}$-submodule $I_{B}$ of $V_{\mathscr{L}}$ is a two-sided graded ideal of the $\mathbb{N}$-graded vertex algebra $V_{\mathscr{L}}$. The $\mathbb{N}$-graded vertex algebra $V_{B}$ associated with the vertex $A$-algebroid $B$ is defined to be the quotient vertex algebra

$$
V_{B}=V_{\mathscr{L}} / I_{B} .
$$

Proposition 2.10 [Gorbounov et al. 2004; Li and Yamskulna 2005]. Let A be a unital commutative associative algebra with identity $e$ and let $B$ be a vertex $A$ algebroid. Then $V_{B}$ is an $\mathbb{N}$-graded vertex algebra such that $\left(V_{B}\right)_{(0)}=A,\left(V_{B}\right)_{(1)}=$ $B$ and for $n \geq 1$,

$$
\begin{aligned}
\left(V_{B}\right)_{(n)}=\operatorname{span}\left\{b_{1}\left(-n_{1}\right) \cdots b_{k}\left(-n_{k}\right) \mathbf{1} \mid b_{i} \in B, n_{1} \geq n_{2} \geq \cdots \geq\right. & n_{k} \geq 1, \\
& \left.n_{1}+\cdots+n_{k}=n\right\} .
\end{aligned}
$$

In particular, $V_{B}$ is generated by the subspace $A \oplus B$.

Next, we discuss homomorphisms and automorphisms for 1-truncated conformal algebras, vertex $A$-algebroids and for the $\mathbb{N}$-graded vertex algebras $V_{B}$.

Definition 2.11. Let $C=A \oplus B$ and $C^{\prime}=A^{\prime} \oplus B^{\prime}$ be 1-truncated conformal algebras. A homomorphism from $C$ to $C^{\prime}$ is a linear map $f: C \rightarrow C^{\prime}$ such that $f(A) \subset A^{\prime}, f(B) \subset B^{\prime}, f \partial=\partial f$, and such that

$$
f\left(u_{i} v\right)=f(u)_{i} f(v)
$$

for $u, v \in C$ and $i=0,1$.

Lemma 2.12. Let $f$ be an endomorphism of a 1-truncated conformal algebra $C=A \oplus B$. Then the linear endomorphism of $L(A \oplus B)$ defined by

$$
\hat{f}\left(u \otimes t^{n}\right)=f(u) \otimes t^{n}
$$

for $u \in A \oplus B$ and $n \in \mathbb{Z}$ gives rise to an endomorphism of $\mathscr{L}$, which we denote by $\hat{f}$ again. Furthermore, $\hat{f}$ preserves the $\mathbb{Z}$-grading of $\mathscr{L}$.

Proof. Using the property that $f \partial=\partial f$, we have $\hat{f} \hat{\partial}=\hat{\partial} \hat{f}$. For $u, v \in C=A \oplus B$, as $f\left(u_{i} v\right)=f(u)_{i} f(v)$ for $i=0,1$, from (2-1)-(2-4) we have

$$
\hat{f}\left(\left[u \otimes t^{m}, v \otimes t^{n}\right]\right)=\left[f(u) \otimes t^{m}, f(v) \otimes t^{n}\right]=\left[\hat{f}\left(u \otimes t^{m}\right), \hat{f}\left(v \otimes t^{n}\right)\right] .
$$


Thus $\hat{f}$ gives rise to an endomorphism of the Lie algebra $\mathscr{L}$. It is clear that $\hat{f}$ preserves the $\mathbb{Z}$-grading.

Definition 2.13. Let $A$ and $A^{\prime}$ be unital commutative associative algebras and let $B$ be a vertex $A$-algebroid, $B^{\prime}$ a vertex $A^{\prime}$-algebroid. A vertex algebroid homomorphism from $B$ to $B^{\prime}$ is a linear map $f: A \oplus B \rightarrow A^{\prime} \oplus B^{\prime}$ such that $f(A) \subset A^{\prime}$, and $f(B) \subset B^{\prime}$ and such that

1. $\left.f\right|_{A}$ is an associative algebra homomorphism.

2. $\left.f\right|_{B}$ is a Leibniz algebra homomorphism.

3. $f(a b)=f(a) f(b)$ for $a \in A$ and $b \in B$.

4. $\langle f(u), f(v)\rangle=f(\langle u, v\rangle)$ for $u, v \in B$.

5. $f \circ \partial=\partial \circ f$.

6. $f\left(b_{0} a\right)=f(b)_{0} f(a)$ for $a \in A$ and $b \in B$.

An automorphism of a vertex A-algebroid $B$ is a bijective vertex algebroid endomorphism of the vertex $A$-algebroid $B$.

Let $(V, Y, \mathbf{1})$ be a vertex algebra. An endomorphism of $V$ is a linear map $g$ : $V \rightarrow V$ such that

$$
\begin{aligned}
g(\mathbf{1}) & =\mathbf{1}, \\
g(Y(u, x) v) & =Y(g(u), x) g(v)
\end{aligned}
$$

for $u, v \in V$. An automorphism of $V$ is a bijective endomorphism of $V$. The group of automorphisms of $V$ is denoted by $\operatorname{Aut}(V)$. If $V=\bigsqcup_{m \in \mathbb{Z}} V_{(m)}$ is a $\mathbb{Z}$ (or $\mathbb{N}$ )-graded vertex algebra, we denote by $\operatorname{Aut}^{0}(V)$ the group of grading-preserving automorphisms of $V$.

Lemma 2.14. Let $B$ be a vertex A-algebroid and let $g$ be a grading-preserving automorphism of the vertex algebra $V_{B}$. Then $g$ restricted to $A \oplus B$ is an automorphism of the vertex A-algebroid $B$.

Proof. As $\left(V_{B}\right)_{(0)}=A$ and $\left(V_{B}\right)_{(1)}=B, g$ is a linear bijection on $A \oplus B$ that preserves the subspaces $A$ and $B$. For $a, a^{\prime} \in A$ and $b, b^{\prime} \in B$, we have

$$
\begin{aligned}
& g\left(a a^{\prime}\right)=g\left(a(-1) a^{\prime}\right)=g(a)_{-1} g\left(a^{\prime}\right)=g(a) g\left(a^{\prime}\right), \\
& g(a b)=g(a(-1) b)=g(a)_{-1} g(b)=g(a) g(b), \\
& g\left(\left[b, b^{\prime}\right]\right)=g\left(b_{0} b^{\prime}\right)=g(b)_{0} g\left(b^{\prime}\right)=\left[g(b), g\left(b^{\prime}\right)\right], \\
& g\left(\left\langle b, b^{\prime}\right\rangle\right)=g\left(b_{1} b^{\prime}\right)=g(b)_{1} g\left(b^{\prime}\right)=\left\langle g(b), g\left(b^{\prime}\right)\right\rangle, \\
& g\left(b_{0} a\right)=g(b)_{0} g(a), \\
& g(\partial(a))=g(a(-2) \mathbf{1})=g(a)_{-2} \mathbf{1}=\partial(g(a)) .
\end{aligned}
$$

Thus $g$ is an automorphism of vertex $A$-algebroid $B$. 
On the other hand, we are going to prove that any automorphism of a vertex $A$ algebroid $B$ extends uniquely to an automorphism of the $\mathbb{N}$-graded vertex algebra $V_{B}$. First we have:

Lemma 2.15. Let $C=A \oplus B$ be a 1-truncated conformal algebra and let $g$ be an endomorphism of $C$. Then $g$ extends uniquely to an endomorphism of the $\mathbb{N}$-graded vertex algebra $V_{\mathscr{L}}$. Furthermore, if $g$ is an automorphism, then the extension is an automorphism.

Proof. Since $A \oplus B$ generates $V_{\mathscr{L}}$ as a vertex algebra, the uniqueness is clear. It remains to prove the existence. By Lemma 2.12, we have a grading-preserving endomorphism $\hat{g}$ of the Lie algebra $\mathscr{L}$, hence a grading-preserving endomorphism of the universal enveloping algebra $U(\mathscr{L})$. Clearly, $\hat{g}$ preserves the Lie subalgebra $\mathscr{L}^{<0}$ and its universal enveloping algebra $U\left(\mathscr{L}^{<0}\right)$. It follows from the construction of $V_{\mathscr{L}}$ that there exists a linear endomorphism $\bar{g}$ of $V_{\mathscr{L}}$ such that $\bar{g}(\mathbf{1})=\mathbf{1}$ and

$$
\bar{g}\left(u_{n} v\right)=g(u)_{n} \bar{g}(v)
$$

for $u \in A \oplus B, v \in V_{\mathscr{L}}, n \in \mathbb{Z}$. Since $V_{\mathscr{L}}$ is generated by $A \oplus B$, it follows form [Lepowsky and Li 2004] that $\bar{g}$ is an endomorphism of $V_{\mathscr{L}}$. Clearly $\bar{g}$ extends $g$.

If $g$ is an automorphism of the 1-truncated conformal algebra $C=A \oplus B$, from the first assertion we have vertex algebra endomorphisms $\bar{g}$ and $\overline{g^{-1}}$ of $V_{\mathscr{L}}$, extending $g$ and $g^{-1}$, respectively. Since $g g^{-1}=g^{-1} g=1$ on $A \oplus B$ and since $A \oplus B$ generates $V_{\mathscr{L}}$ as a vertex algebra, we have $\bar{g} \overline{g^{-1}}=\overline{g^{-1}} \bar{g}=1$. Thus, $\bar{g}$ is an automorphism of $V_{\mathscr{L}}$.

Proposition 2.16. Let $g$ be an endomorphism of a vertex A-algebroid B. Then $g$ extends uniquely to an endomorphism of $V_{B}$ as an $\mathbb{N}$-graded vertex algebra. Furthermore, if $g$ is an automorphism, then the extension is an automorphism.

Proof. The uniqueness is clear, as $A \oplus B$ generates $V_{B}$ as a vertex algebra. For the existence, first by Lemma 2.15, we have a grading-preserving endomorphism $\bar{g}$ of the vertex algebra $V_{\mathscr{L}}$, extending $g$. Now we show that $\bar{g}$ reduces to an endomorphism of $V_{B}$. Recall that $V_{B}=V_{\mathscr{L}} / I_{B}$, where $I_{B}$ is the two-sided ideal of $V_{\mathscr{L}}$, generated by

$$
E=\operatorname{span}\left\{e-\mathbf{1}, a(-1) a^{\prime}-a a^{\prime}, a(-1) b-a b \mid a, a^{\prime} \in A, b \in B\right\} .
$$

Now, we must prove $\bar{g}\left(I_{B}\right) \subset I_{B}$. As $E$ generates $I_{B}$ as a two-sided ideal, it suffices to prove that $\bar{g}(E) \subset E$. Let $a, a^{\prime} \in A, b \in B$. We have

$$
\begin{aligned}
& \bar{g}(e-\mathbf{1})=e-\mathbf{1} \in E, \\
& \bar{g}\left(a(-1) a^{\prime}-a a^{\prime}\right)=g(a)(-1) g\left(a^{\prime}\right)-g(a) g\left(a^{\prime}\right) \in E, \\
& \bar{g}(a(-1) b-a b)=g(a)(-1) g(b)-g(a) g(b) \in E .
\end{aligned}
$$


This proves $\bar{g}(E) \subset E$. Therefore, $\bar{g}$ reduces to an endomorphism of the $\mathbb{N}$-graded vertex algebra $V_{B}$. The second assertion follows immediately from the proof of the second assertion of Lemma 2.15.

Combining Lemma 2.14 with Proposition 2.16, we have:

Theorem 2.17. Let $A$ be a unital commutative associative algebra and let $B$ be a vertex A-algebroid. The group $\operatorname{Aut}^{0}\left(V_{B}\right)$ of grading-preserving automorphisms of the $\mathbb{N}$-graded vertex algebra $V_{B}$ is isomorphic to the group of automorphisms of vertex $A$-algebroid $B$ with the restriction map as an isomorphism.

\section{Classification of graded simple twisted $V_{B}$-modules}

In this section we construct and classify graded simple twisted $V_{B}$-modules by exploiting a twisted analogue of the Lie algebra $\mathscr{L}$. First, we recall the definition of the notion of twisted module for a vertex algebra and we discuss several properties of twisted modules.

Let $V$ be a vertex algebra and let $g$ be an automorphism of $V$ of order $T<\infty$. Decompose $V$ into eigenspaces of $g$ :

$$
V=\coprod_{r=0}^{T-1} V^{r}, \quad \text { where } V^{r}=\left\{v \in V \mid g(v)=e^{2 r \pi \sqrt{-1} / T} v\right\} .
$$

A g-twisted $V$-module [Lepowsky 1985; Frenkel et al. 1988; Feingold et al. 1991; Dong 1994] is a vector space $M$ equipped with a linear map

$$
\begin{aligned}
Y_{M}: V & \rightarrow(\text { End } M) \llbracket x^{1 / T}, x^{-1 / T} \rrbracket, \\
u & \mapsto Y_{M}(u, x)=\sum_{n \in(1 / T) \mathbb{Z}} u_{n} x^{-n-1}
\end{aligned}
$$

satisfying the following conditions:

1. For $u \in V, w \in M$, we have $u_{n} w=0$ for $n \in \frac{1}{T} \mathbb{Z}$ sufficiently large.

2. $Y_{M}(\mathbf{1}, x)=1_{M}$ (the identity operator on $\left.M\right)$.

3. For $u \in V^{r}$ with $0 \leq r \leq T-1$,

$$
Y_{M}(u, x)=\sum_{n \in(r / T)+\mathbb{Z}} u_{n} x^{-n-1} \in x^{-r / T}(\text { End } M) \llbracket x, x^{-1} \rrbracket .
$$

4. For $u \in V^{r}$ with $0 \leq r \leq T-1, v \in V$,

$$
\begin{array}{r}
x_{0}^{-1} \delta\left(\frac{x_{1}-x_{2}}{x_{0}}\right) Y_{M}\left(u, x_{1}\right) Y_{M}\left(v, x_{2}\right)-x_{0}^{-1} \delta\left(\frac{x_{2}-x_{1}}{-x_{0}}\right) Y_{M}\left(v, x_{2}\right) Y_{M}\left(u, x_{1}\right) \\
=x_{2}^{-1}\left(\frac{x_{1}-x_{0}}{x_{2}}\right)^{-r / T} \delta\left(\frac{x_{1}-x_{0}}{x_{2}}\right) Y_{M}\left(Y\left(u, x_{0}\right) v, x_{2}\right)
\end{array}
$$

(the twisted Jacobi identity). 
Remark 3.1. Let $\left(M, Y_{M}\right)$ be a $g$-twisted $V$-module and let $U$ be any vertex subalgebra of $V^{0}$. Then $M$ is a $U$-module. Thus, if $g$ is taken to be the identity map, the notion of $g$-twisted $V$-module reduces to that of $V$-module while the twisted Jacobi identity reduces to the ordinary (untwisted) Jacobi identity.

Lemma 3.2 ([Dong et al. 1998]; compare [Dong et al. 1997]). Let $\left(M, Y_{M}\right)$ be a g-twisted $V$-module. Then

$$
Y_{M}(\mathscr{D} v, x)=\frac{d}{d x} Y_{M}(v, x)
$$

for $v \in V$, where $\mathscr{D} v=v_{-2} \mathbf{1}$.

Remark 3.3. For $v \in V, u \in V^{r}, p \in \mathbb{Z}$ and $s, t \in \mathbb{Q}$, extracting the coefficients of $z_{0}^{-p-1} z_{1}^{-s-1} z_{2}^{-t-1}$ from the twisted Jacobi identity (3-2) we get

$$
\begin{aligned}
\sum_{m \geq 0}\left(\begin{array}{c}
s \\
m
\end{array}\right)\left(u_{p+m} v\right)_{s+t-m} & \\
& =\sum_{m \geq 0}(-1)^{m}\left(\begin{array}{c}
p \\
m
\end{array}\right)\left\{u_{p+s-m} v_{t+m}-(-1)^{p} v_{p+t-m} u_{s+m}\right\} .
\end{aligned}
$$

By taking $\operatorname{Res}_{x_{0}}$ of (3-2), we obtain the twisted commutator formula:

$$
\begin{aligned}
& {\left[Y_{M}\left(u, x_{1}\right), Y_{M}\left(v, x_{2}\right)\right]} \\
& \quad=\operatorname{Res}_{x_{0}} x_{2}^{-1}\left(\frac{x_{1}-x_{0}}{x_{2}}\right)^{-r / T} \delta\left(\frac{x_{1}-x_{0}}{x_{2}}\right) Y_{M}\left(Y\left(u, x_{0}\right) v, x_{2}\right) .
\end{aligned}
$$

Multiplying (3-2) by $\left(\frac{x_{1}-x_{0}}{x_{2}}\right)^{r / T}$ and then taking $\operatorname{Res}_{x_{1}}$, we obtain the twisted iterate formula:

$$
Y_{M}\left(Y\left(u, x_{0}\right) v, x_{2}\right)=\operatorname{Res}_{x_{1}}\left(\frac{x_{1}-x_{0}}{x_{2}}\right)^{r / T} \cdot X
$$

where

$X=x_{0}^{-1} \delta\left(\frac{x_{1}-x_{2}}{x_{0}}\right) Y_{M}\left(u, x_{1}\right) Y_{M}\left(v, x_{2}\right)-x_{0}^{-1} \delta\left(\frac{x_{2}-x_{1}}{-x_{0}}\right) Y_{M}\left(v, x_{2}\right) Y_{M}\left(u, x_{1}\right)$.

From the twisted Jacobi identity one has the following twisted weak associativity: For $u \in V^{r}$ with $0 \leq r \leq T-1$ and for $v \in V, w \in W$,

$$
\left(x_{0}+x_{2}\right)^{k+(r / T)} Y_{M}\left(u, x_{0}+x_{2}\right) Y_{M}\left(v, x_{2}\right) w=\left(x_{2}+x_{0}\right)^{k+(r / T)} Y_{M}\left(Y\left(u, x_{0}\right) v, x_{2}\right) w
$$

where $k$ is a nonnegative integer such that $x^{k+(r / T)} Y_{M}(u, x) w \in M \llbracket x \rrbracket$. One can prove, as in [Li 1996, Lemma 2.8] that the twisted Jacobi identity is equivalent to the twisted commutator formula and the twisted weak associativity. 
Let $M$ be a $g$-twisted $V$-module. For a subset $U$ of $M$, denote the smallest $g$-twisted $V$-submodule containing $U$ by $\langle U\rangle$, which is called the $g$-twisted $V$ submodule generated by $U$. Just as with untwisted modules, from the twisted weak associativity, we have

$$
\langle U\rangle=\operatorname{span}\left\{v_{n} w \mid v \in V, n \in \frac{1}{T} \mathbb{Z}, w \in U\right\} .
$$

We define

$$
\operatorname{Ann}_{V}(U)=\{v \in V \mid Y(v, x) w=0 \quad \text { for } w \in U\},
$$

the annihilator of $U$ in $V$.

Proposition 3.4. For any subset $U$ of a g-twisted $V$-module $M$, the annihilator $\operatorname{Ann}_{V}(U)$ is an ideal of $V$. Moreover,

$$
\operatorname{Ann}_{V}(U)=\operatorname{Ann}_{V}(\langle U\rangle) .
$$

Proof. This follows from the proof of [Lepowsky and Li 2004, Proposition 4.5.11], with weak associativity and weak commutativity replaced by twisted associativity and twisted commutativity.

Let $S$ be a subset of $V$. Define

$$
\operatorname{Ann}_{M}(S)=\left\{w \in M \mid Y_{M}(v, x) w=0 \text { for } v \in S\right\},
$$

the annihilator of $S$ in $M$. We follow [Lepowsky and Li 2004]. By suitably modifying the proof of Proposition 4.5.14 of that reference, replacing weak commutativity by twisted commutativity and replacing Proposition 4.5 .11 by Proposition 3.4 of the present work, we have:

Proposition 3.5. For a subset $S$ of $V$, the annihilator $\operatorname{Ann}_{M}(S)$ is a g-twisted $V$-submodule of $M$. Furthermore,

$$
\operatorname{Ann}_{M}(S)=\operatorname{Ann}_{M}(\langle S\rangle) .
$$

Here $\langle S\rangle$ is the ideal of $V$ generated by $S$.

Lemma 3.6 [Li 1996, Lemma 2.11]. Let $V$ be a vertex algebra with an automorphism $g$ of order $T$ and let $a \in V^{k}$ and $b, u^{0}, \ldots, u^{r} \in V$ with $0 \leq k \leq T-1$. If

$$
\left[Y\left(a, x_{1}\right), Y\left(b, x_{2}\right)\right]=\sum_{j=0}^{r} \frac{1}{j !} Y\left(u^{j}, x_{2}\right)\left(\frac{\partial}{\partial x_{2}}\right)^{j} x_{1}^{-1} \delta\left(\frac{x_{2}}{x_{1}}\right)
$$

acting on $V$, then for any g-twisted $V$-module $\left(M, Y_{M}\right)$ we have

$$
\left[Y_{M}\left(a, x_{1}\right), Y_{M}\left(b, x_{2}\right)\right]=\sum_{j=0}^{r} \frac{1}{j !} Y_{M}\left(u^{j}, x_{2}\right)\left(\frac{\partial}{\partial x_{2}}\right)^{j} x_{1}^{-1} \delta\left(\frac{x_{2}}{x_{1}}\right)\left(\frac{x_{2}}{x_{1}}\right)^{k / T}
$$


acting on M. On the other hand, the converse is also true for any faithful g-twisted $V$-module $\left(M, Y_{M}\right)$.

Definition 3.7. Let $V=\bigsqcup_{m \in \mathbb{Z}} V_{(m)}$ be a $\mathbb{Z}$-graded vertex algebra. A $\frac{1}{T} \mathbb{N}$-graded $g$-twisted $V$-module is a $g$-twisted $V$-module $M$ equipped with a $\frac{1}{T} \mathbb{N}$-grading

$$
M=\coprod_{n \in(1 / T) \mathbb{N}} M(n)
$$

such that

$$
v_{m} M(n) \subset M(n+p-m-1)
$$

for $v \in V_{(p)}$ and $m, n \in \frac{1}{T} \mathbb{Z}$ with $p \in \mathbb{Z}$.

Next, we study $\frac{1}{T} \mathbb{N}$-graded $g$-twisted modules for the $\mathbb{N}$-graded vertex algebra $V_{B}$ associated to a vertex $A$-algebroid $B$, where $g$ is an automorphism of order $T<$ $\infty$ of the $\mathbb{N}$-graded vertex algebra $V_{B}$ and of the vertex $A$-algebroid $B$ (compare Theorem 2.17).

Noticing that $A \oplus B$ is a 1-truncated conformal algebra, we start with a general 1-truncated conformal algebra $C=C_{0} \oplus C_{1}$ with an automorphism $g$ of $C$ of order $T<\infty$. Associated with the 1 -truncated conformal algebra $C=C_{0} \oplus C_{1}$ we have the Lie algebra $\mathscr{L}(C)$ and the vertex algebra $V_{\mathscr{L}(C)}$ with $C$ as a generating subspace. In view of Lemma $2.15 \mathrm{~g}$ is an order- $T$ automorphism of the vertex algebra $V_{\mathscr{L}(C)}$.

Lemma 3.8 ([Dong et al. 1998]; compare [Borcherds 1986]). Let $V$ be a vertex algebra and let $T$ be a positive integer. Set

$$
L_{T}(V)=V \otimes \mathbb{C}\left[t^{1 / T}, t^{-1 / T}\right],
$$

a vector space, and set

$$
\hat{\partial}=\mathscr{D} \otimes 1+1 \otimes \frac{d}{d t},
$$

a linear operator on $L_{T}(V)$. The bilinear (multiplicative) operation on $L_{T}(V)$, defined by

$$
\left[u \otimes t^{m}, v \otimes t^{n}\right]=\sum_{i \geq 0}\left(\begin{array}{c}
m \\
i
\end{array}\right)\left(u_{i} v \otimes t^{m+n-i}\right)
$$

for $u, v \in V, m, n \in \frac{1}{T} \mathbb{Z}$, gives rise to a Lie algebra structure on $L_{T}(V) / \hat{\partial} L_{T}(V)$, which is denoted by $\mathscr{L}(V, T)$. Furthermore, any order-T automorphism $g$ of $C$ gives rise to an order-T automorphism, also denoted by $g$, of $\mathscr{L}(V, T)$, where

$$
g\left(v \otimes t^{n}\right)=e^{-2 n \pi \sqrt{-1}}\left(g v \otimes t^{n}\right)
$$

for $v \in V$ and $n \in \frac{1}{T} \mathbb{Z}$. 
Specializing Lemma 3.8 with $V=V_{\mathscr{L}(C)}$, we have a Lie algebra $\mathscr{L}\left(V_{\mathscr{L}(C)}, T\right)$ and an automorphism $g$. For $u \in C$ and $m \in \frac{1}{T} \mathbb{Z}$, denote by $u(m)$ the canonical image of $u \otimes t^{m}$ in $\mathscr{L}\left(V_{\mathscr{L}(C)}, T\right)$. We have

$$
\begin{aligned}
(\partial a)(m) & =-m a(m-1), \\
{[u(m), v(n)] } & =\sum_{i=0}^{1}\left(\begin{array}{c}
m \\
i
\end{array}\right)\left(u_{i} v\right)(m+n-i)
\end{aligned}
$$

for $a \in C_{0}, u, v \in C$ and $m, n \in \frac{1}{T} \mathbb{Z}$. Because $u_{i} v \in C$ for $u, v \in C$ and $i \geq 0$, we see that the $u(m)$, for $u \in C$ and $m \in \frac{1}{T} \mathbb{Z}$, span a Lie subalgebra $\mathscr{L}(C, T)$ of $\mathscr{L}\left(V_{\mathscr{L}(C)}, T\right)$. Denote by $\mathscr{L}(C, g)$ the $g$-fixed point Lie subalgebra:

$$
\mathscr{L}(C, g)=\mathscr{L}(C, T)^{g} .
$$

Using Lemma 3.8 we immediately have:

Proposition 3.9. Let $C=C_{0} \oplus C_{1}$ be a 1-truncated conformal algebra and let $g$ be an order- $T$ automorphism of $C$. Then

$$
\mathscr{L}(C, g)=L(C, g) / \hat{\partial} L\left(C_{0}, g\right),
$$

as a vector space, where

$$
L(C, g)=\coprod_{r=0}^{T-1} C^{r} \otimes t^{r / T} \mathbb{C}\left[t, t^{-1}\right],
$$

$L\left(C_{0}, g\right)$ is a subspace defined in the obvious way, and $\hat{\partial}$ is given by

$$
\hat{\partial}=\partial \otimes 1+1 \otimes d / d t: L\left(C_{0}, g\right) \rightarrow L(C, g) .
$$

For $u \in C^{r}$ with $0 \leq r \leq T-1$ and for $n \in \mathbb{Z}$, denote by $u(n+r / T)$ the canonical image of $u \otimes t^{n+r / T}$ in $\mathscr{L}(C, g)$. Then the following relations hold for $a \in C_{0}^{r}$, $a^{\prime} \in C_{0}^{r^{\prime}}, b \in C_{1}^{s}, b^{\prime} \in C_{1}^{s^{\prime}}, m, n \in \mathbb{Z}$ :

$$
\begin{aligned}
(\partial a)\left(m+\frac{r}{T}\right) & =-\left(m+\frac{r}{T}\right) a\left(m-1+\frac{r}{T}\right) \\
{\left[a\left(m+\frac{r}{T}\right), a^{\prime}\left(n+\frac{r^{\prime}}{T}\right)\right] } & =0, \\
{\left[a\left(m+\frac{r}{T}\right), b\left(n+\frac{s}{T}\right)\right] } & =\left(a_{0} b\right)\left(m+n+\frac{r+s}{T}\right), \\
{\left[b\left(m+\frac{s}{T}\right), b^{\prime}\left(n+\frac{s^{\prime}}{T}\right)\right] } & =\left(b_{0} b^{\prime}\right)\left(m+n+\frac{s+s^{\prime}}{T}\right) \\
& +\left(m+\frac{s}{T}\right)\left(b_{1} b^{\prime}\right)\left(m+n+\frac{s+s^{\prime}}{T}-1\right) .
\end{aligned}
$$


We define

$$
\begin{array}{ll}
\operatorname{deg} a(n+r / T)=-n-1 & \text { for } a \in C_{0}^{r}, n \in \mathbb{Z}, \\
\operatorname{deg} b(n+r / T)=-n & \text { for } b \in C_{1}^{r}, n \in \mathbb{Z},
\end{array}
$$

making $\mathscr{L}(C, g)$ a $\frac{1}{T} \mathbb{Z}$-graded Lie algebra. For $n \in \frac{1}{T} \mathbb{Z}$, denote by $\mathscr{L}(C, g)_{(n)}$ the degree- $n$ subspace. We have the following triangular decomposition

$$
\mathscr{L}(C, g)=\mathscr{L}(C, g)_{+} \oplus \mathscr{L}(C, g)_{(0)} \oplus \mathscr{L}(C, g)_{-},
$$

where $\mathscr{L}(C, g)_{ \pm}=\bigsqcup_{0<n \in \frac{1}{T} \mathbb{Z}} \mathscr{L}(C, g)_{( \pm n)}$. Notice that $\mathscr{L}(C, g)_{(0)}$ is spanned by the elements $a(-1), b(0)$ for $a \in C_{0}^{0}$ and $b \in C_{1}^{0}$.

For $u \in C^{r}$ with $0 \leq r \leq T-1$, form the generating function

$$
u(x)=\sum_{n \in \frac{r}{T}+\mathbb{Z}} u(n) x^{-n-1} \in \mathscr{L}(C, g) \llbracket x^{1 / T}, x^{-1 / T} \rrbracket .
$$

For any $\mathscr{L}(C, g)$-module $W$, consider $u(x)$ as an element of (End $W) \llbracket x^{1 / T}, x^{-1 / T} \rrbracket$, which we denote by $u_{W}(x)$ :

$$
u_{W}(x)=u(x)=\sum_{n \in(1 / T) \mathbb{Z}} u(n) x^{-n-1} \in(\text { End } W) \llbracket x^{1 / T}, x^{-1 / T} \rrbracket .
$$

Lemma 3.10. The commutation relations (3-10)-(3-12) amount to the following relations in terms of generating functions:

$$
\begin{aligned}
{\left[a\left(x_{1}\right), a^{\prime}\left(x_{2}\right)\right] } & =0, \\
{\left[a\left(x_{1}\right), b^{\prime}\left(x_{2}\right)\right] } & =x_{2}^{-1}\left(\frac{x_{1}}{x_{2}}\right)^{-r / T} \delta\left(\frac{x_{1}}{x_{2}}\right)\left(a_{0} b^{\prime}\right)\left(x_{2}\right), \\
{\left[b\left(x_{1}\right), b^{\prime}\left(x_{2}\right)\right] } & =x_{2}^{-1}\left(\frac{x_{1}}{x_{2}}\right)^{-r / T} \delta\left(\frac{x_{1}}{x_{2}}\right)\left(b_{0} b^{\prime}\right)\left(x_{2}\right) \\
& +\left(b_{1} b^{\prime}\right)\left(x_{2}\right) \frac{\partial}{\partial x_{2}} x_{2}^{-1}\left(\frac{x_{1}}{x_{2}}\right)^{-r / T} \delta\left(\frac{x_{1}}{x_{2}}\right)
\end{aligned}
$$

for $a \in C_{0}^{r}, b \in C_{1}^{r}, a^{\prime} \in C_{0}$, and $b^{\prime} \in C_{1}$. Moreover, we have

$$
(\partial a)(x)=\frac{d}{d x} a(x) \quad \text { for } a \in C_{0} .
$$

From these relations we immediately have:

Corollary 3.11. For $a, a^{\prime} \in C_{0}$ and $b, b^{\prime} \in C_{1}$,

$$
\begin{aligned}
{\left[a\left(x_{1}\right), a^{\prime}\left(x_{2}\right)\right] } & =0, \\
\left(x_{1}-x_{2}\right)\left[a\left(x_{1}\right), b\left(x_{2}\right)\right] & =0, \\
\left(x_{1}-x_{2}\right)^{2}\left[b\left(x_{1}\right), b^{\prime}\left(x_{2}\right)\right] & =0 .
\end{aligned}
$$


Definition 3.12. An $\mathscr{L}(C, g)$-module $W$ is said to be restricted if for any $w \in W$ and $u \in C^{r}$ with $0 \leq r \leq T-1, u(n+r / T) w=0$ for $n \in \mathbb{Z}$ sufficiently large, that is, $u_{W}(x) \in \operatorname{Hom}\left(W, W\left(\left(x^{1 / T}\right)\right)\right)$ for $u \in C$.

The following result is analogous to a result of [Li 1996] for twisted affine Lie algebras:

Proposition 3.13. Let $C=C_{0} \oplus C_{1}$ be a 1-truncated conformal algebra and let $g$ be an automorphism of $V_{\mathscr{L}(C)}$ of order $T$, which is extended from an automorphism of $C$. Every $g$-twisted $V_{\mathscr{L}(C)}$-module $W$ is naturally a restricted $\mathscr{L}(C, g)$-module with $u_{W}(x)=Y_{W}(u, x)$ for $u \in C$. Moreover, the set of $g$-twisted $V_{\mathscr{L}(C)}$-submodules of $W$ is precisely the set of $\mathscr{L}(C, g)$-submodules of $W$. On the other hand, for any restricted $\mathscr{L}(C, g)$-module $W$, there exists a unique g-twisted $V_{\mathscr{L}(C)}$-module structure $Y_{W}$ on $W$ such that

$$
Y_{W}(u, x)=u_{W}(x) \quad \text { for } u \in C=C_{0} \oplus C_{1} \subset V_{\mathscr{L}(C)} .
$$

Proof. On the vertex algebra $V_{\mathscr{L}(C)}$, the following relations hold for $a, a^{\prime} \in C_{0}$ and $b, b^{\prime} \in C_{1}$ :

$$
\begin{aligned}
& {\left[Y\left(a, x_{1}\right), Y\left(a^{\prime}, x_{2}\right)\right]=0,} \\
& {\left[Y\left(a, x_{1}\right), Y\left(b^{\prime}, x_{2}\right)\right]=x_{2}^{-1} \delta\left(\frac{x_{1}}{x_{2}}\right) Y\left(a_{0} b^{\prime}, x_{2}\right),} \\
& {\left[Y\left(b, x_{1}\right), Y\left(b^{\prime}, x_{2}\right)\right]=x_{2}^{-1} \delta\left(\frac{x_{1}}{x_{2}}\right) Y\left(b_{0} b^{\prime}, x_{2}\right)+Y\left(b_{1} b^{\prime}, x_{2}\right) \frac{\partial}{\partial x_{2}} x_{2}^{-1} \delta\left(\frac{x_{1}}{x_{2}}\right) .}
\end{aligned}
$$

From Lemmas 3.6 and 3.10, every $g$-twisted $V_{\mathscr{L}(C)}$-module $W$ is naturally a restricted $\mathscr{L}(C, g)$-module with $u_{W}(x)=Y_{W}(u, x)$ for $u \in C$. As $C$ generates $V_{\mathscr{L}(C)}$ as a vertex algebra, the set of $g$-twisted $V_{\mathscr{L}(C)}$-submodules of $W$ is precisely the set of $\mathscr{L}(C, g)$-submodules of $W$.

Let $S=\operatorname{span}\left\{u_{W}(x) \mid u \in C\right\}$. In view of Corollary 3.11, $S$ is a local subspace of $\operatorname{Hom}\left(W, W\left(\left(x^{1 / T}\right)\right)\right)$. Note that $\operatorname{Hom}\left(W, W\left(\left(x^{1 / T}\right)\right)\right)$ is naturally $\mathbb{Z} / T \mathbb{Z}$-graded:

$$
\operatorname{Hom}\left(W, W\left(\left(x^{1 / T}\right)\right)\right)=\coprod_{r=0}^{T-1} x^{r / T} \operatorname{Hom}(W, W((x))),
$$

and that $S$ is a graded subspace.

Let $\sigma_{T}$ be the linear automorphism of $\operatorname{Hom}\left(W, W\left(\left(x^{1 / T}\right)\right)\right)$ defined by

$$
\sigma_{T}(\alpha(x))=e^{-2 r \pi \sqrt{-1} / T} \alpha(x)
$$

for $\alpha(x) \in x^{r / T} \operatorname{Hom}(W, W((x)))$ with $0 \leq r \leq T-1$; compare (3-1).

$S$ generates a vertex algebra $\langle S\rangle$ inside $\operatorname{Hom}\left(W, W\left(\left(x^{1 / T}\right)\right)\right)$ [Li 1996] with the identity operator $1_{W}$ as the vacuum vector and with $\sigma_{T}$ as an automorphism. $W$ is 
naturally a faithful $\sigma_{T}$-twisted $\langle S\rangle$-module with $Y_{W}\left(\alpha(x), x_{0}\right)=\alpha\left(x_{0}\right)$. With the relations (3-15)-(3-17), by Lemma 3.6, we have

$\left[Y\left(a_{W}(x), x_{1}\right), Y\left(a_{W}^{\prime}(x), x_{2}\right)\right]=0$,

$\left[Y\left(a_{W}(x), x_{1}\right), Y\left(b_{W}^{\prime}(x), x_{2}\right)\right]=x_{2}^{-1} \delta\left(\frac{x_{1}}{x_{2}}\right) Y\left(\left(a_{0} b^{\prime}\right)_{W}(x), x_{2}\right)$,

$\left[Y\left(b_{W}(x), x_{1}\right), Y\left(b_{W}^{\prime}(x), x_{2}\right)\right]=x_{2}^{-1} \delta\left(\frac{x_{1}}{x_{2}}\right) Y\left(\left(b_{0} b^{\prime}\right)_{W}(x), x_{2}\right)$

$$
+Y\left(\left(b_{1} b^{\prime}\right)_{W}(x), x_{2}\right) \frac{\partial}{\partial x_{2}} x_{2}^{-1} \delta\left(\frac{x_{1}}{x_{2}}\right)
$$

for $a \in C_{0}^{r}, b \in C_{1}^{r}, a^{\prime} \in C_{0}$, and $b^{\prime} \in C_{1}$. We also have

$$
Y\left((\partial a)_{W}(x), x_{1}\right)=Y\left(\frac{d}{d x} a_{W}(x), x_{1}\right)=\frac{\partial}{\partial x_{1}} Y\left(a_{W}(x), x_{1}\right)
$$

for $a \in A$. By Lemmas 3.6 and 3.10, $\langle S\rangle$ is naturally an $\mathscr{L}(C)$-module with $u_{\langle S\rangle}\left(x_{1}\right)=Y\left(u_{W}(x), x_{1}\right)$ for $u \in C$. Furthermore, $\langle S\rangle$ as an $\mathscr{L}(C)$-module is generated by $1_{W}$ and we have $u_{W}(x)_{n} 1_{W}=0$ for $u \in C, n \geq 0$. From the construction of $V_{\mathscr{L}(C)}$ as an $\mathscr{L}(C)$-module, there exists a unique $\mathscr{L}(C)$-module homomorphism $\psi$ from $V_{\mathscr{L}(C)}$ to $\langle S\rangle$, sending 1 to $1_{W}$. As $V_{\mathscr{L}(C)}$ as a vertex algebra is generated by $C, \psi$ is a vertex algebra homomorphism. We have

$$
\psi(u)=\psi(u(-1) \mathbf{1})=u_{W}(x)_{-1} 1_{W}=u_{W}(x)
$$

for $u \in C$. It is clear that $\psi\left(C^{r}\right) \subset S^{r}$ for $0 \leq r \leq T-1$. As $C$ generates $V_{\mathscr{L}(C)}$ as a vertex algebra, $\psi$ preserves the $\mathbb{Z} / T \mathbb{Z}$-gradings, i.e., $\sigma_{T} \psi=\psi g$. Consequently, $W$ is a $g$-twisted $V_{\mathscr{L}(C)}$-module.

For the rest of this paper, we assume that $A$ is a unital commutative associative algebra whose identity is denoted by $e$ and $B$ is a vertex $A$-algebroid and we assume that $g \in \operatorname{Aut}^{0}\left(V_{B}\right)$ with $o(g)=T<\infty$. Recall that $C=A \oplus B$ is naturally a 1 -truncated conformal algebra. An $\mathscr{L}(C, g)$-module of level $k \in \mathbb{C}$ is an $\mathscr{L}(C, g)$ module on which $e(-1)$ acts as scalar $k$.

Immediately from Proposition 3.13 we have:

Proposition 3.14. Every $g$-twisted $V_{B}$-module is naturally a restricted $\mathscr{L}(C, g)$ module of level 1 . Moreover, the set of $g$-twisted $V_{B}$-submodules is precisely the set of $\mathscr{L}(C, g)$-submodules.

We have the following decompositions into $g$-eigenspaces:

$$
V_{B}=\coprod_{r=0}^{T-1} V_{B}^{r}, \quad A=\coprod_{r=0}^{T-1} A^{r}, \quad B=\coprod_{r=0}^{T-1} B^{r} .
$$


Clearly, $A^{0}$ is a subalgebra of $A$, containing the identity, and $B^{0}$ is a vertex $A^{0}$ algebroid. Furthermore, by Lemma $2.6, B^{0} / A^{0} \partial A^{0}$ is a Lie $A^{0}$-algebroid. Set

$$
I=\sum_{r=1}^{T-1} A^{r} \cdot A^{T-r} \subset A^{0} .
$$

It is clear that $I$ is a two-sided ideal of $A^{0}$, so that $A^{0} / I$ is a unital commutative associative algebra. Furthermore, $B^{0} /\left(I \cdot B^{0}+A^{0} \partial A^{0}\right)$ is a Lie $A^{0} / I$-algebroid.

Proposition 3.15. Let $M=\bigsqcup_{n \in(1 / T) \mathbb{N}} M(n)$ be a $\frac{1}{T} \mathbb{N}$-graded g-twisted $V_{B}$-module. Then $M(0)$ is a module for the Lie $A^{0}$-algebroid $B^{0} / A^{0} \partial A^{0}$ with

$$
\begin{array}{ll}
a \cdot w=a_{-1} w & \text { for } a \in A^{0}, w \in M(0), \\
b \cdot w=b_{0} w & \text { for } b \in B^{0}, w \in M(0) .
\end{array}
$$

Furthermore, for $a \in A^{r}, a^{\prime} \in A^{T-r}, b \in B^{T-r}$ with $0<r \leq T-1$ and for $w \in M(0)$, we have $\left(a a^{\prime}\right) \cdot w=0$ and $(a b) \cdot w=\left(1-\frac{r}{T}\right)\left(a_{0} b\right) \cdot w$.

Proof. Let $U$ be the vertex subalgebra of $V_{B}$ generated by $A^{0} \oplus B^{0}$. As $A^{0} \oplus B^{0} \subset$ $V_{B}^{0}, U$ is actually a vertex subalgebra of $V_{B}^{0}$. From Remark 3.1, $M$ is a $U$-module. With $\left(V_{B}\right)_{(0)}=A$ and $\left(V_{B}\right)_{(1)}=B$, we have $\left(V_{B}^{0}\right)_{(0)}=A^{0}$ and $\left(V_{B}^{0}\right)_{(1)}=B^{0}$. Consequently, we have $U_{(0)}=A^{0}$ and $U_{(1)}=B^{0}$. It follows from the construction of $V_{B^{0}}$ that $U$ is a homomorphic image of the vertex algebra $V_{B^{0}}$, so that $W$ is naturally a $V_{B^{0}}$-module. By [Li and Yamskulna 2005, Proposition 4.8], $W(0)$ is naturally a module for the Lie $A^{0}$-algebroid $B^{0} / A^{0} \partial A^{0}$.

Let $a \in A^{r}, a^{\prime} \in A^{T-r}, b \in B^{T-r}, w \in M(0)$ with $0<r \leq T-1$. By substituting $u=a, v=a^{\prime}, p=-1, s=-1+\frac{r}{T}, t=-\frac{r}{T}$ in (3-4), we get

$$
\begin{aligned}
\left(a a^{\prime}\right) \cdot w & =\left(a a^{\prime}\right)_{-1} w=\left(a(-1) a^{\prime}\right)_{-1} w \\
& =\sum_{m \geq 0} a_{-2+(r / T)-m} a_{-(r / T)+m}^{\prime} w+a_{-1-(r / T)-m}^{\prime} a_{-1+(r / T)+m} w=0 .
\end{aligned}
$$

Similarly, by substituting $u=a, v=b, p=-1, s=-1+\frac{r}{T}$ and $t=1-\frac{r}{T}$ in (3-4), we have

$$
\begin{aligned}
(a b) \cdot w & =(a b)_{0} w=(a(-1) b)_{0} w \\
= & \left(1-\frac{r}{T}\right)\left(a_{0} b\right)_{-1} w \\
& \quad+\sum_{m \geq 0}\left\{a_{-2+(r / T)-m} b_{1-(r / T)+m}+b_{-(r / T)-m} a_{-1+(r / T)+m}\right\} w \\
& =\left(1-\frac{r}{T}\right)\left(a_{0} b\right) \cdot w,
\end{aligned}
$$

completing the proof. 
Let $U$ be a module for the Lie $A^{0}$-algebroid $B^{0} / A^{0} \partial A^{0}$ such that

$$
\left(a a^{\prime}\right) \cdot u=0 \quad \text { and } \quad(a b) \cdot u=\left(1-\frac{r}{T}\right)\left(a_{0} b\right) \cdot u
$$

for $a \in A^{r}, a^{\prime} \in A^{T-r}, b \in B^{T-r}, u \in U$, and $0<r \leq T-1$. We construct a $\frac{1}{T} \mathbb{N}$ graded $g$-twisted $V_{B}$-module $M=\bigsqcup_{n \in(n / T) \mathbb{N}} M(n)$ with $M(0)=U$ as a module for the Lie $A^{0}$-algebroid $B^{0} / A^{0} \partial A^{0}$.

First, $U$ is a module for the Lie algebra $A^{0} \oplus B^{0} / \partial A^{0}$. Recall that $\mathscr{L}(C, g)_{(0)}=$ $A^{0} \oplus B^{0} / \partial A^{0}$. For convenience, we set $\mathscr{L}(C, g)_{\leq 0}=\mathscr{L}(C, g)_{(0)} \oplus \mathscr{L}(g)_{-}$. Then $U$ is an $\mathscr{L}(C, g)_{\leq 0}$-module under the actions

$$
\begin{aligned}
a\left(n+\frac{r}{T}-1\right) \cdot u & =\delta_{n+r /(T), 0} a u, \\
b\left(n+\frac{r}{T}\right) \cdot u & =\delta_{n+r /(T), 0} b u
\end{aligned}
$$

for $a \in A^{r}, b \in B^{r}$, and $n \geq 0$. Next, we form the induced $\mathscr{L}(C, g)$-module

$$
M_{g}(U)=\operatorname{Ind}_{\mathscr{L}(C, g)_{\leq 0}}^{\mathscr{L}(C, g)} U=U(\mathscr{L}(C, g)) \otimes_{U\left(\mathscr{L}(C, g)_{\leq 0}\right)} U .
$$

We endow $U$ with degree 0 , making $M_{g}(U)$ a $\frac{1}{T} \mathbb{N}$-graded restricted $\mathscr{L}(C, g)$ module. By Proposition 3.13, $M_{g}(U)$ is naturally a $g$-twisted $V_{\mathscr{L}(C)}$-module. In view of the P-B-W theorem, we may and we should consider $U$ as the degree-zero subspace of $M_{g}(U)$.

We set

$$
W_{g}(U)=\operatorname{span}\left\{v_{n} u \mid v \in E, n \in \frac{1}{T} \mathbb{Z}, u \in U\right\} \subset M_{g}(U)
$$

and define

$$
M_{B}(U)=M_{g}(U) / U(\mathscr{L}(C, g)) W_{g}(U) .
$$

Since $U(\mathscr{L}(C, g)) W_{g}(U)$ is an $\mathscr{L}(C, g)$-submodule of $M_{g}(U)$, by Proposition 3.13 $U(\mathscr{L}(C, g)) W_{g}(U)$ is a $g$-twisted $V_{\mathscr{L}(C)}$-submodule. Then $M_{B}(U)$ is a $g$-twisted $V_{\mathscr{L}(C)}$-module. Clearly, $M_{B}(U)$ is generated by $\bar{U}$ the image of $U$ in $M_{B}(U)$. In fact, $M_{B}(U)$ is a $g$-twisted $V_{B}$-module by the following:

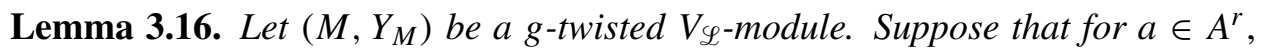
$a^{\prime} \in A, b \in B$ with $0 \leq r \leq T-1$, we have

$$
\begin{aligned}
Y_{M}(e, x) w & =w, \\
Y_{M}\left(a(-1) a^{\prime}, x\right) w & =Y_{M}\left(a a^{\prime}, x\right) w, \\
Y_{M}(a(-1) b, x) w & =Y_{M}(a b, x) w
\end{aligned}
$$

for all $w \in K$, where $K$ is a generating subspace of $M$. Then $M$ is naturally a g-twisted $V_{B}$-module. 
Proof. Recall that

$$
E=\operatorname{span}\left\{e-1, a(-1) a^{\prime}-a a^{\prime}, a(-1) b-a b \mid a, a^{\prime} \in A, b \in B\right\} \subset V_{\mathscr{L}(C)} .
$$

By the assumptions on $a, a^{\prime}, b$, we have $K \subset \operatorname{Ann}_{M}(E)$. Using Proposition 3.5, we obtain $\operatorname{Ann}_{M}\left(I_{B}\right)=\operatorname{Ann}_{M}(E)$. Since $\operatorname{Ann}_{M}\left(I_{B}\right)$ is a $g$-twisted $V_{\mathscr{L}(C)}$ submodule of $M$ and $M$ is generated by $K$, we have $\operatorname{Ann}_{M}\left(I_{B}\right)=M$. This implies that $M$ is a $g$-twisted $V_{B}$-module.

One can see that Lemma 3.16 indeed implies that $M_{B}(U)$ is naturally a $g$-twisted $V_{B}$-module. Furthermore we have:

Theorem 3.17. Let $U$ be a module for the Lie $A^{0}$-algebroid $B^{0} / A^{0} \partial A^{0}$ such that

$$
\left(a a^{\prime}\right) \cdot u=0 \quad \text { and }(a b) \cdot u=\left(1-\frac{r}{T}\right)\left(a_{0} b\right) \cdot u
$$

for $a \in A^{r}, a^{\prime} \in A^{T-r}, b \in B^{T-r}, u \in U, r \neq 0$. Then $M_{B}(U)$ is naturally $a$ g-twisted $V_{B}$-module such that $M_{B}(U)(0)=U$.

Proof. To show that $M_{B}(U)(0)=U$, we must prove that $\left(U(\mathscr{L}(g)) W_{g}(U)\right)(0)=0$. First we show that $W_{g}(U)(0)=0$. Notice that for $v \in\left(V_{\mathscr{L}(C)}\right)_{(m)}^{r}$ with $m \in \mathbb{Z}$, we have deg $v_{k+r / T}=m-k-r / T-1$ for $k \in \mathbb{Z}$. Then from the definition of $W_{g}(U)$, $W_{g}(U)(0)$ is spanned by the vectors

$$
(e-\mathbf{1})_{-1} u, \quad\left(a(-1) a^{\prime}\right)_{-1} u-\left(a a^{\prime}\right)_{-1} u, \quad(a(-1) b)_{0} u-(a b)_{0} u
$$

for $u \in U, a \in A^{r}, a^{\prime} \in A^{T-r}, b \in B^{T-r}$ with $0 \leq r \leq T-1$. Since $e_{-1}$ acts as $e$ (the identity of $A^{0}$ ) on $U$, we have $(e-\mathbf{1})_{-1} u=0$ for $u \in U$. If $r=0$, by (3-4),

$$
\left(a_{-1} a^{\prime}\right)_{-1} u=a(-1) a^{\prime}(-1) u=a\left(a^{\prime} u\right)=\left(a a^{\prime}\right) u=\left(a a^{\prime}\right)_{-1} u,
$$

and

$$
(a(-1) b)_{0} u=a(-1) b(0) u=a(b u)=(a b) u=(a b)_{0} u .
$$

Next, we assume that $r>0$. By (3-4), we have

$$
\begin{aligned}
& \left(a(-1) a^{\prime}\right)_{-1} u \\
& \quad=\sum_{i=0}^{\infty} a\left(-1-i+\frac{r}{T}\right) a^{\prime}\left(i-1-\frac{r}{T}\right) u+\sum_{i=0}^{\infty} a^{\prime}\left(-2-i-\frac{r}{T}\right) a\left(i+\frac{r}{T}\right) u \\
& =a\left(-1+\frac{r}{T}\right) a^{\prime}\left(-1-\frac{r}{T}\right) u=a^{\prime}\left(-1-\frac{r}{T}\right) a\left(-1-\frac{r}{T}\right) u \\
& =0 \\
& =\left(a a^{\prime}\right)_{-1} u
\end{aligned}
$$


and

$$
\begin{aligned}
& (a(-1) b)_{0} u \\
& =\sum_{i=0}^{\infty} a\left(-1-i+\frac{r}{T}\right) b\left(i-\frac{r}{T}\right) u+\sum_{i=0}^{\infty} b\left(-i-1-\frac{r}{T}\right) a\left(i+\frac{r}{T}\right) u-\frac{r}{T}\left(a_{0} b\right)_{-1} u \\
& =a\left(-1+\frac{r}{T}\right) b\left(-\frac{r}{T}\right) u-\frac{r}{T}\left(a_{0} b\right) \cdot u \\
& \quad=b\left(-\frac{r}{T}\right) a\left(-1+\frac{r}{T}\right) u+\left(a_{0} b\right)_{-1} u-\frac{r}{T}\left(a_{0} b\right)_{-1} u \\
& =\left(1-\frac{r}{T}\right)\left(a_{0} b\right) \cdot u \\
& =(a b) \cdot u .
\end{aligned}
$$

Hence, $W_{g}(U)(0)=0$.

We now show that

$$
\mathscr{L}(C, g)_{\leq 0} W_{g}(U) \subset W_{g}(U) .
$$

Recall from [Li and Yamskulna 2005, Lemma 4.2] that

$$
v_{i} E \subset E \quad \text { for } v \in C=A \oplus B, i \geq 0 .
$$

Since $M_{g}(U)$ is a $\frac{1}{T} \mathbb{N}$-graded $\mathscr{L}(C, g)$-module with $U$ as the degree-zero subspace, we have $\mathscr{L}(C, g)_{\leq 0} U \subset U$. For $v \in C=A \oplus B, c \in E, m, t \in \frac{1}{T} \mathbb{Z}, u \in U$, from the twisted commutator formula (3-5) (cf. (3-8)), we have

$$
v_{m} c_{t} u=c_{t} v_{m} u+\sum_{i \geq 0}\left(\begin{array}{c}
m \\
i
\end{array}\right)\left(v_{i} c\right)_{m-t-i} u .
$$

These immediately imply that $\mathscr{L}(C, g)_{\leq 0} W_{g}(U) \subset W_{g}(U)$. Then

$$
\begin{aligned}
U(\mathscr{L}(C, g)) W_{g}(U) & =U\left(\mathscr{L}(C, g)_{+}\right) U\left(\mathscr{L}(C, g)_{\leq 0}\right) W_{g}(U) \\
& =U\left(\mathscr{L}(C, g)_{+}\right) W_{g}(U) \\
& =W_{g}(U)+\mathscr{L}(C, g)_{+} U\left(\mathscr{L}(C, g)_{+}\right) W_{g}(U),
\end{aligned}
$$

which implies that $\left(U(\mathscr{L}(C, g)) W_{g}(U)\right)(0)=0$. This completes the proof.

Next, we continue with Theorem 3.17 to construct and classify $\frac{1}{T} \mathbb{N}$-graded simple $g$-twisted $V_{B}$-modules. Let $U$ be a module for the Lie $A^{0}$-algebroid $B^{0} / A^{0} \partial A^{0}$ as in Theorem 3.17. Let $J(U)$ be the sum of all graded $\mathscr{L}(C, g)$-submodules of $M_{g}(U)$ with trivial degree-zero subspaces. Then $J(U)$ is the unique maximal graded $\mathscr{L}(C, g)$-submodule of $M_{g}(U)$ with the property that $J(U) \cap U=0$. Set

$$
L_{g}(U)=M_{g}(U) / J(U),
$$

a $\frac{1}{T} \mathbb{N}$-graded $g$-twisted $V_{B}$-module. 
Lemma 3.18. Let $U$ be a module for the Lie $A^{0}$-algebroid $B^{0} / A^{0} \partial A^{0}$ as in Theorem 3.17. Then $L_{g}(U)$ is a $\frac{1}{T} \mathbb{N}$-graded $g$-twisted $V_{B}$-module such that $L_{g}(U)(0)=$ $U$ as a module for the Lie $A^{0}$-algebroid $B^{0} / A^{0} \partial A^{0}$ and such that for any nonzero graded submodule $W$ of $L_{g}(U)$, we have $W(0) \neq 0$. Furthermore, if $U$ is a simple $B^{0} / A^{0} \partial A^{0}$-module, $L_{g}(U)$ is a $\frac{1}{T} \mathbb{N}$-graded simple g-twisted $V_{B}$-module.

Proof. Similar to the proof of Theorem 4.12 in [Li and Yamskulna 2005].

Lemma 3.19. Let $W=\bigsqcup_{n \in(1 / T) \mathbb{Z}} W(n)$ be a $\frac{1}{T} \mathbb{N}$-graded simple g-twisted $V_{B^{-}}$ module with $W(0) \neq 0$. Then $W \cong L_{g}(W(0))$.

Proof. Similar to the proof of Lemma 4.13 in [Li and Yamskulna 2005].

To summarize we have:

Theorem 3.20. Let $H$ be a complete set of equivalence class representatives of simple modules for the Lie $A^{0}$-algebroid $B^{0} / A^{0} \partial A^{0}$ satisfying the condition that

$$
\left(a a^{\prime}\right) U=0,\left((a b)-\left(1-\frac{r}{T}\right)\left(a_{0} b\right)\right) U=0
$$

for $a \in A^{r}, a^{\prime} \in A^{T-r}, b \in B^{T-r}$ with $0<r<T$. Then $\left\{L_{g}(U) \mid U \in H\right\}$ is a complete set of equivalence class representatives of $\frac{1}{T} \mathbb{N}$-graded simple g-twisted $V_{B}$-modules.

Proof. Similar to the proof of Theorem 4.14 in [Li and Yamskulna 2005].

Finally, we remark that by taking $g=1$, the identity map of $V_{B}$, we recover Theorem 4.14 of [Li and Yamskulna 2005]:

Corollary 3.21. If $H$ is a complete set of equivalence class representatives of sim-

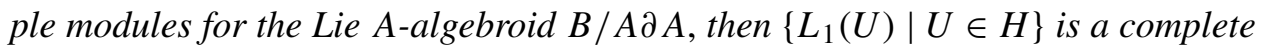
set of equivalence class representatives of $\mathbb{N}$-graded simple $V_{B}$-modules.

\section{References}

[Berman et al. 2002a] S. Berman, Y. Billig, and J. Szmigielski, "Vertex operator algebras and the representation theory of toroidal algebras", pp. 1-26 in Recent developments in infinite-dimensional Lie algebras and conformal field theory, edited by S. Berman et al., Contemp. Math. 297, Amer. Math. Soc., Providence, RI, 2002. MR 2003j:17037 Zbl 1018.17017

[Berman et al. 2002b] S. Berman, C. Dong, and S. Tan, "Representations of a class of lattice type vertex algebras”, J. Pure Appl. Algebra 176:1 (2002), 27-47. MR 2003k:17034 Zbl 1043.17016

[Borcherds 1986] R. E. Borcherds, "Vertex algebras, Kac-Moody algebras, and the Monster", Proc. Nat. Acad. Sci. U.S.A. 83:10 (1986), 3068-3071. MR 87m:17033 Zbl 0613.17012

[Bressler 2002] P. Bressler, "Vertex algebroids, I", preprint, 2002. math.AG/0202185

[Bressler 2003] P. Bressler, "Vertex algebroids, II", preprint, 2003. math.AG/0304115

[Dong 1994] C. Dong, "Twisted modules for vertex algebras associated with even lattices", J. Algebra 165:1 (1994), 91-112. MR 95i:17032 Zbl 0807.17023 
[Dong et al. 1997] C. Dong, H. Li, and G. Mason, "Regularity of rational vertex operator algebras", Adv. Math. 132:1 (1997), 148-166. MR 98m:17037 Zbl 0902.17014

[Dong et al. 1998] C. Dong, H. Li, and G. Mason, “Twisted representations of vertex operator algebras", Math. Ann. 310:3 (1998), 571-600. MR 99d:17030 Zbl 0890.17029

[Dong et al. 2002] C. Dong, H.-S. Li, and G. Mason, "Vertex Lie algebra, vertex Poisson algebras and vertex algebras", pp. 69-96 in Recent developments in infinite-dimensional Lie algebras and conformal field theory (Charlottesville, VA, 2000), edited by S. Berman et al., Contemp. Math. 297, Amer. Math. Soc., Providence, RI, 2002. MR 2003h:17035

[Fĕgin and Frenkel 1988] B. L. Fĕ̌gin and È. V. Frenkel, "A family of representations of affine Lie algebras", Uspekhi Mat. Nauk 43:5 (1988), 227-228. In Russian; translated in Russian Math. Surveys 43 (1988), 221-222. MR 89k:17016 Zbl 0668.17015

[Fer̆gin and Frenkel 1990a] B. L. Fer̆gin and E. V. Frenkel, "Affine Kac-Moody algebras and semiinfinite flag manifolds", Comm. Math. Phys. 128 (1990), 161-189. MR 92f:17026 Zbl 0722.17019

[Feigin and Frenkel 1990b] B. L. Feigin and E. V. Frenkel, "Representations of affine Kac-Moody algebras and bosonization", pp. 271-316 in Physics and mathematics of strings, edited by L. Brink et al., World Sci., Teaneck, NJ, 1990. MR 92d:17025 Zbl 0734.17011

[Feingold et al. 1991] A. J. Feingold, I. B. Frenkel, and J. F. X. Ries, Spinor construction of vertex operator algebras, triality, and $E_{8}^{(1)}$, Contemporary Mathematics 121, Amer. Math. Soc., Providence, RI, 1991. MR 92k:17041 Zbl 0743.17029

[Frenkel and Ben-Zvi 2001] E. Frenkel and D. Ben-Zvi, Vertex algebras and algebraic curves, Mathematical Surveys and Monographs 88, Amer. Math. Soc., Providence, RI, 2001. MR 2003f:17036 Zbl 0981.17022

[Frenkel et al. 1988] I. Frenkel, J. Lepowsky, and A. Meurman, Vertex operator algebras and the Monster, Pure and Applied Mathematics 134, Academic Press, Boston, 1988. MR 90h:17026 Zbl 0674.17001

[Gorbounov et al. 2004] V. Gorbounov, F. Malikov, and V. Schechtman, "Gerbes of chiral differential operators, II: Vertex algebroids”, Invent. Math. 155:3 (2004), 605-680. MR 2005e:17047 Zbl 1056.17022

[Lepowsky 1985] J. Lepowsky, "Calculus of twisted vertex operators", Proc. Nat. Acad. Sci. U.S.A. 82:24 (1985), 8295-8299. MR 88f:17030 Zbl 0579.17010

[Lepowsky and Li 2004] J. Lepowsky and H. Li, Introduction to vertex operator algebras and their representations, Progress in Mathematics 227, Birkhäuser, Boston, 2004. MR 2004k:17050 Zbl 1055.17001

[Li 1996] H.-S. Li, "Local systems of twisted vertex operators, vertex operator superalgebras and twisted modules", pp. 203-236 in Moonshine, the Monster, and related topics (South Hadley, MA, 1994), edited by C. Dong and G. Mason, Contemp. Math. 193, Amer. Math. Soc., Providence, RI, 1996. MR 96m:17050 Zbl 0844.17022

[Li and Yamskulna 2005] H. Li and G. Yamskulna, "On certain vertex algebras and their modules associated with vertex algebroids”, J. Algebra 283:1 (2005), 367-398. MR 2006h:17035 Zbl 1066.17017

[Malikov and Schechtman 1999] F. Malikov and V. Schechtman, "Chiral de Rham complex, II", preprint, 1999. math.AG/9901065

[Malikov et al. 1998] F. Malikov, V. Schechtman, and A. Vaintrob, "Chiral de Rham complex", preprint, 1998. math.AG/9803041

[Wakimoto 1986] M. Wakimoto, "Fock representations of the affine Lie algebra $A_{1}^{(1), ", C o m m . ~ M a t h . ~}$ Phys. 104:4 (1986), 605-609. MR 87m:17011 Zbl 0587.17009 
Received January 13, 2006.

\section{HAISHENG LI}

Department of Mathematical SCIEnCES

RUTGERS UNIVERSITY

311 N. 5TH STREET

CAMDEN, NJ 08102

UNITED STATES

and

DEPARTMENT OF MATHEMATICS

HARBIN NORMAL UNIVERSITY

HARBIN

CHINA

hli@camden.rutgers.edu

GAYWALEE YAMSKULNA

DEPARTMENT OF MATHEMATICS

ILLINOIS STATE UNIVERSITY

CAMPUS BOX 4520

NORMAL, IL 61790-4520

UNITED STATES

and

INSTITUTE OF SCIENCE

WALAILAK UNIVERSITY

NAKHON SI THAMMARAT

THAILAND

gyamsku@ilstu.edu 